\title{
Análisis de la traducción de I Viceré de De Roberto (Los Virreyes) por José R. Monreal
}

\section{Fernando Molina Castillo}

Universidad de Sevilla

fmolina@siff.us.es

La editorial Acantilado (Barcelona, 2008), en su línea de difusión en España de la gran literatura de todos los tiempos y países, ha publicado una traducción española de I Vicerè de De Roberto, a cargo de José Ramón Monreal. En 1994, en el centenario de la novela, ${ }^{1}$ ya vieron la luz en nuestro país otras dos traducciones: una del mismo Monreal (Madrid, Anaya \& Mario Muchnik) y otra de María Teresa Navarro para las Letras Universales, de Cátedra. Aquella primera traducción de Monreal ha sido revisada antes de ser de nuevo publicada en Acantilado, de manera que ésta puede ser considerada una nueva traducción.

Entre la abundante literatura traducida, tanto italiana (Salgari, Nievo), ${ }^{2}$ como no italiana (Chateaubriand, Chesterfield, Maupassant, Stevenson, Twain, Balzac, Zola, Flaubert, etc...), Monreal ha mostrado predilección por los autores sicilianos: Capuana (El marqués de Roccaverdina, Barcelona, Azar, 2003), Pirandello (El turno, Barcelona, Ed. del Bronce, 1999; La tragedia de un personaje, Barcelona, Acantilado, 2002; Uno, ninguno y cien mil, id., 2004), Tomasi di Lampedusa (Conversaciones literarias, Barcelona, Bruguera, 1983) y Sciascia (La bruja y el capitán, Barcelona, Tusquets, 1987; reed., 2006; 1912+1, id. 2001), a los cuales hay que añadir al napolitano, aunque siciliano de adopción — de Catania, para más señas_ - De Roberto, de quien ha traducido también los Processi verbali (Atestados, Madrid, Anaya \& Mario Muchnik, 1995).

No es ésta la ocasión para hacer un análisis crítico de la novela, para lo cual remitimos, como punto de partida, a la introducción de M. T. Navarro en la edición ya citada, con bibliografía (p. 9-69). ${ }^{3}$ Tampoco es mi intención, por

1. La $1^{\mathrm{a}}$ ed. vio la luz en Milán: Galli di C. Chiesa e F. Guindani, 1894. La 2a ed. lo hizo veintiséis años más tarde, en 1920 (Milán: Fratelli Treves), revisada por el autor. La ed. a cargo de Z. Nunzio (Milán: Rizzoli, 1998), repropone el texto de esta 2a ed.

2. De la reciente traducción de Le Confessioni d’un Italiano (Barcelona: Acantilado, 2008), pionera y única en España, nos ocupamos también en este mismo número.

3. Añado algunas referencias de estudios destacables sobre I Viceré posteriores a esa fecha: Gli inganni del romanzo. I Viceré tra storia e finzione letteraria (Atti del Congresso celebrativo del centenario dei "Viceré», Catania, 23-26 nov. 1994), Catania: Fondazione Verga, 1998; M. POlaCCO, «I Vicerè (1894)», en F. BerTONi; D. Giglioli (a cura di), Quindici episodi 
razones de espacio y de oportunidad, analizar a fondo la traducción de Navarro (en adelante $\mathrm{N}$, seguido del número de página), aunque obviamente la tendré presente y recurriré a ella para contrastar las soluciones adoptadas por Monreal (en adelante M) que iré comentando.

Respecto a la versión de $1994^{4}$ (en adelante M 1994), el traductor ha llevado a cabo una profunda revisión de índole estilística, en pequeños detalles repartidos en cada página, dando lugar a un texto mejorado, más fluido y preciso. Son frecuentes las modificaciones del imperfecto subjuntivo (estuviese por estuviera, osase por atreviera, etc...), la evitación del leísmo (cogerle por cogerlo, le reclamaba por lo reclamaba, etc...), una colocación menos forzada de los pronombres reflexivos (tratábase por se trataba), y, en general, un mayor acierto en la elección de las palabras y fórmulas expresivas para traducir, algo que sería interminable de ejemplificar. Un aspecto concreto que ha modificado es el de los pronombres de tratamiento, de los cuales nos ocuparemos más abajo.

Por otra parte — aunque posiblemente esté dictado por criterios marcados por la editorial - no habría estado de más mantener el breve ensayo de Sciascia «Por qué Croce no tenía razón», incluido en M 1994, y en general, algún soporte crítico mínimo, que rebasara el ámbito de las solapas y la contraportada. También habría sido bueno mantener el cuadro genealógico de los Uzeda que aparecía en la ed. 1994 (p. 14), útil para que el lector se oriente entre la colmena de personajes de una novela larga y compleja. Y puestos a sugerir, sería muy útil que alguna edición aportara una lista de personajes, al estilo de la que aporta M. Armiño en su monumental traducción del Tiempo perdido de Proust.

El traductor muestra una amplia capacidad para afrontar terminologías específicas, que abundan en la novela, relativas, por ejemplo, al campo de las finanzas y la economía, el derecho, la heráldica, etc..., así como la fraseología italiana. También es acertada la introducción de cambios en la puntuación (ej. p. 63), creando párrafos más breves que contribuyen a oxigenar un texto original en ocasiones excesivamente denso, y de ese modo, agilizar su lectura.

del romanzo italiano (1881-1923), Bolonia: Pendragon, 1999, p. 149-174; M. GANERI, «La svolta dei Viceré», en Belfagor, LIV, 1999, 539-48; S. DAI PRA, Federico De Roberto, tra naturalismo ed espressionismo: lo stile della provocazione, Palermo: Istituto Siciliano di studi politici ed economici, 2003; M. GANERI, L'Europa in Sicilia. Saggi su Federico De Roberto, Florencia: Le Monnier, 2005. Ya en fase de corrección de pruebas de este trabajo, conocí, dentro del citado volumen Gli inganni del romanzo, el artículo "Tradurre la lingua dei Viceré: il modello spagnolo» (p. 487-525), de María T. Navarro (a quien agradezco su diligencia en facilitarme una copia), en el que se analizan y contraponen las dos traducciones de la novela publicadas en 1994, la propia de Navarro y la de Monreal. He constatado que, salvo muy escasas excepciones, los errores de traducción (en sus distintos grados de gravedad) que Navarro detecta en M 1994, muy numerosos, siguen apareciendo en la versión nueva, de 2008. Por otra parte, dichas críticas a la traducción de M 1994 señaladas por Navarro apenas coinciden con las que yo recojo de la nueva versión y comento en este trabajo, de manera que puede decirse que ambos artículos, el de Navarro y éste, lejos de solaparse, se suman, reforzando así la consideración de que la traducción de Monreal, en cualquiera de sus dos versiones, no puede considerarse, en rigor, buena.

4. Un amplio cotejo entre las dos versiones de Monreal ha sido realizado por María Jesús Carrasco, cuya colaboración agradezco. 
No obstante, y más aun tratándose de un texto extenso, la traducción no está exenta de pasajes puntuales que, a mi modo de ver, no reflejan de la mejor manera posible el texto original. A partir de una lectura atenta del original ${ }^{5}$ y la traducción, en este comentario se expondrán y analizarán dichos pasajes —en su mayoría puntuales y aislados - sin otro afán que el de la colaboración y la curiosidad intelectuales, y el de la búsqueda de la precisión filológica, así como el de la reflexión sobre la traducción en sí. La reseña aspira a ser así una aportación que propongo, modestamente, para una eventual futura revisión del trabajo.

\section{I}

En primer lugar comentaré los que, a mi juicio, constituyen errores de traducción achacables a una interpretación equivocada del original, ya sea de una determinada palabra en un contexto dado, o bien de una estructura sintáctica más o menos compleja.

En «Intanto la notizia dell'arrivo del duca s'era subito diffusa per la città, e le prime visite gli furono annunziate che egli non s'era neppur riposato del viaggio» (I, II; p. 45), «le prime visite» es sujeto, mientras que el duque ( $g l i)$ es el destinatario, o complemento indirecto, del anuncio. Por tanto, la traducción «y a las primeras visitas se les anunció que ni siquiera había descansado del viaje» (M 51) es errónea. El equívoco posiblemente nace del «che» polivalente, que en este caso equivale a "quando", con el que el narrador imita un registro hablado de la lengua. La traducción debería ser más bien: «y las primeras visitas le fueron anunciadas [al duque] cuando aún no había descansado del viaje» (o bien, cfr. N 120: «y cuando todavía no había descansado del viaje, llegaron los anuncios de las primeras visitas»).

En el testamento de la princesa Teresa (I, II; p. 51), se lee: «[...] tali e tante passività oberavano la sostanza del mio consorte, che essa poteva considerarsi, anzi era effettivamente distrutta ed alla vigilia di venire smembrata tra i molteplici suoi creditori». Debemos entender que "la sostanza», es decir, «el patrimonio», estaba muy próximo, o a punto de verse repartido entre sus muchos acreedores, y no que ya estuviera «en vísperas de mi llegada, repartido en manos de sus muchos acreedores» (M 57). Cfr. N 127: «en vísperas de ser desmembrada entre los numerosos acreedores».

En I, II (p. 42) leemos: «Il presidente era sicuro, checché si dicesse in contrario, che l'erede sarebbe stato il principe, con un forte legato al conte». Como también en español, legado es un término jurídico que designa «una disposición que en su testamento o codicilo hace un testador a favor de una o varias personas» (DRAE). La frase quiere decir, por tanto, que «el heredero iba a ser el

5. Para el texto original de la novela he utilizado y cito de la edición publicada en Milán: Mondadori, 1991, con introducción de V. Spinazzola, contrastándola con la ed. a cargo de G. Giudice, F. De Roberto, I Viceré e altre opere, Turín: UTET, 1982, así como la de C. A. Madrignani, F. De Roberto, Romanzi, novelle e saggi, Milán: Mondadori, 1984 (empleada como base del texto para el cd-rom Letteratura Italiana Zanichelli 4.0, $4^{\mathrm{a}} \mathrm{ed}$, 2001). Los subrayados en todas las citas, del original y las traducciones, son míos. 
príncipe, con una considerable manda para el conde» (N 117) y no «en estrecha unión con el conde» (M 47).

En «Lodovico era stato quasi soppresso per dar posto a Raimondo» (I, III; p. 70), se alude al hecho de que Lodovico, siendo mayor que Raimondo, prácticamente había sido suprimido del testamento a favor de Raimondo (N 148: «casi había sido anulado»). Leemos, sin embargo, que «Lodovico se había visto como sorprendido de tener que ceder su puesto a Raimondo» (M 77).

En la descripción del cuchitril en el que vive Ferdinando, en la Pietra d'Ovo, notamos dos nuevos errores de interpretación: «Dormiva sopra una specie di cuccetta da marinaio, costruiva da sé tavole e seggiole, e la casa pareva un arsenale dalla tanta roba che v'era sparsa; seghe, [...]; e poi un assortimento di assi e di travi, e sacchi di farina per fare il pane, provviste di polvere, una scansia di libri [...]» (I, III; p. 79). Es decir, además de dormir en una especie de litera de marinero, Ferdinando se construía él mismo sus mesas y sus sillas, y por otra parte, había, entre otras cosas, «sacos de harina para hacer el pan» y "provisiones de pólvora». ${ }^{6} \mathrm{La}$ traducción de $\mathrm{M}$ es la siguiente: «Dormía en una especie de litera de marinero que se había fabricado él mismo con sillas y mesas, y la casa parecía un arsenal, de tantas cosas como había esparcidas por ella; sierras [...], así como toda clase de tablas y de vigas, de sacos de harina para hacer el pan llenos de polvo, una estantería con libros [...]» (M 87).

En I, III (p. 75) leemos que «Il monaco era per questo andato assiduamente dietro ai nipoti, fuorché a Raimondo, al quale non rivolgeva la parola da anni ed anni per la ragione che era stato il beniamino della madre, incitandoli a farsi valere», debiéndose entender que es el monje don Blasco quien incita a los sobrinos a hacerse valer, y no la madre a Raimondo, como se interpreta de M 82: «Y tal era la razón de que el monje hubiera corrido con frecuencia detrás de sus sobrinos, a excepción de Raimondo, al que no dirigía la palabra desde hacía largos años, por el simple hecho de ser el preferido de la madre, quien lo empujaba a hacerse valens.?

En I, VII (p. 208), se describe la actitud de Mara Fersa, suegra de Isabella Pinto al ser ésta la esposa de su hijo Mario, al percibir que entre su nuera y Raimondo Uzeda la amistad estaba dando paso a una mayor intimidad, algo que "non le piaceva nient'affatto e non intendeva che continuasse», que es traducido del siguiente modo: «no por ello le hacía ninguna gracia y no comprendía que la cosa continuase» (M 226). En este contexto intendere no significa tanto «comprender, entender» como «tener la intención». Propongo «no estaba dispuesta a permitir que la cosa continuase» (cfr. N 296: «tenía la intención de que no continuara».

6. Cfr. N 157-8: «Dormía en una especie de litera de marinero, se construía las mesas y las sillas, y había tantas cosas esparcidas por la casa que parecía un arsenal: sierras [...] y también una selección de tablas y vigas y sacos de harina para hacer pan, acopio de pólvora, un estante de libros $[\ldots]$ ».

7. Cfr. N 152: «el monje había perseguido asiduamente a sus sobrinos, a excepción de Raimondo, al que desde hacía muchos años no le dirigía la palabra, porque había sido el preferido de la madre, incitándoles a que hicieran valer sus derechos». 
«Lucrezia gonfiava, eccitata dalle notizie del trionfo di Giulente, impaziente di tornare in città per rivederlo, irritata dagli sconvenienti motteggi della zia» (I, VIII; p. 262), traducido del siguiente modo: «Lucrezia estaba exultante, excitada por las noticias del triunfo de Giulente, impaciente por volver a la ciudad para verlo e irritada por los impedimentos que le ponía su tía» (M 282). Sin embargo, motteggio es «scherzo o battuta spiritosa» (DISC). En efecto, la tía Ferdinanda se burla de que los partidarios de la Unificación, por ser analfabetos, habían colocado los carteles que decían «sí» al revés, y decía «iNo dicen sí, sino is, chis, chis! ¡Fuera de aquí, chis!». Por tanto, los sconvenienti motteggi son más bien «burlas inoportunas» (N 352), o acaso «impertinentes».

Del duque de Oragua se dice en un panfleto que ya desde 1848 se mostró «largo del suo prezioso ausilio ai liberali» (I, IX; p. 274), donde largo es sinónimo de generoso, como a continuación se detalla: «Vuelca su contribución a la formación de las escuadras de voluntarios, presta ayuda a cuantos liberales perseguidos sufren en la indigencia» (M 295). No es admisible, por tanto, la traducción "Sería largo de contar aquí su inestimable ayuda a los liberales», toda vez que, como es sabido, el it. largo no equivale al esp. "largo», sino a "ancho». ${ }^{8}$

Observemos la expresión «i famigliari del principe» en el siguiente contexto: «La servitù, al palazzo Francalanza, non discorreva d'altro, dimenticava perfino il fidanzamento di Benedetto Giulente con la signorina Lucrezia. Quest'avvenimento [...] aveva già provocado un risveglio dei partiti in cui $i$ famigliari del principe eran divisi; e mentre Giuseppe, il portinaio, si scappellava [...], Pasqualino Riso non si toccava neppure il berretto» (II, I, p. 289). Los famigliari, en mi opinión, tiene en este caso la acepción arcaica de «servidumbre, criados» (N 384: «los sirvientes del príncipe»), atestiguada por GDLI (s. v., \$\$ 9-11), y no la de «familiares» (M 312). Aunque en descargo hay que recordar que «familiar» también tiene en español la acepción marginal de «criado» (DRAE, Moliner).

La expresión «moglie della mano manca» (II, v, p. 384) equivale a «esposa morganática», es decir, de rango inferior al marido por no pertenecer a la realeza o la nobleza; ${ }^{9}$ más genéricamente puede entenderse como «mujer ilegítima» (N 482). Por tanto, no es válida la traducción «mujer con mano izquierda» (M 409), como tampoco lo es la de «Lucrezia, che oramai era tutta una cosa con la cognata della mano manca» (II, IV; p. 362): «Lucrezia, uña y carne ya con su cuñada gracias a la mano izquierda de ésta» (M 387). ${ }^{10}$ En un tercer caso, sin embargo, la traducción de Monreal sí resulta aceptable: «figliuoli della mano manca, anzi di nessuna mano» (I, VI; p. 191), en referencia a los hijos ilegítimos de don Blasco: «hijos tenidos bajo mano, o bajo ninguna» (M 208). ${ }^{11}$

Sólo en determinados contextos, el verbo italiano curare puede traducirse con curar. Es el caso, por ejemplo, de «Se fossero fenomeni isterici, i dottori

8. Cfr. N 366: «Aquí es generoso con su preciosa ayuda [...]».

9. Cfr. GDLI, «Mano», $\$ 10$, ejemplificando precisamente con este pasaje derobertiano.

10. N 461: «Lucrezia, que ya se había convertido en uña y carne de la cuñada por parte ilegítima».

11. N 277: «hijos de la rama torcida o mejor dicho de ninguna rama». 
l'avvrebbero curata» (III, IX; p. 658): «Si fueran fenómenos histéricos, los médicos la habrían curado» (M 688; también «curado» en N 769). En la mayoría de los casos, sin embargo, equivale más bien a tratar, atender, cuidar, ${ }^{12}$ como en «Un brutto giorno donna Teresa, già malandata, si svegliò con un doloretto al fianco, del quale sulle prime non si curò, ma che un anno dopo doveva condurla al sepolcro" (I, III; p. 86), donde non si curò quiere decir que "no prestó atención», o «se despreocupó». ${ }^{13}$ Sin embargo, leemos «Un aciago día doña Teresa, ya con la salud quebrantada, se despertó con un dolorcillo en un costado, del que no sólo no se curaría, sino que había de llevarla un año más tarde a la tumba» (M 94). Más interesante resulta aún el siguiente pasaje, en el que aparecen reunidos ambos verbos: «Lo aveva curato, gli aveva dimostrato il bene fraterno che gli voleva, s'era valsa dell'ascendente che esercitava su lui per guarirlo» (III, VII; p. 613), traducido «Le había curado, le había dado muestras del afecto fraterno que buscaba en ella, se había valido del ascendiente que ejercía sobre él para curarle» (M 643). ${ }^{14}$

Sabida es la polisemia del término paese: puede significar localidad pequeña (pueblo), país o incluso territorio o región. Como es fácil suponer, es una palabra que tiene una alta frecuencia de uso en la novela, en unos casos para referirse al pueblo —o ciudad — de los Uzeda, Catania, en otros para al país entero, Italia. En varios casos $\mathrm{M}$ opta por la segunda acepción en vez de por la primera, cuando en mi opinión - y también la de $\mathrm{N}$ - equivale a pueblo o ciudad:

«Maledetto paese!» (I, VII; p. 212): «iMaldito pais!» (M 230);

«a quest'ora sarei scappato anche a piedi da questo fetido paese» (I, VII; p. 231): «a estas horas me habría largado incluso a pie de este hediondo país» (M 249);

«Vedi [... Come tutto il paese è per lui?» (I, IX; p. 287): «¿Ves que todo el país está con él?» (M 309);

«ricominciò a dirigere nel suo paese e in quasi tutta la Sicilia il movimento contro il regime borbonico» (I, III; p. 108): «volvió a dirigir en su región y en casi toda Sicilia el movimiento contra el régimen borbónico» (M 118).

Incluso, en «Ho lavorato parecchi anni pel mio paese» (III, VIII; p. 642), ambos traductores, M 672 y N 752, optan por «mi país», aunque en mi opinión el personaje Consalvo se está refiriendo a su ciudad, Catania, de cuyo cargo de alcalde está anunciando la dimisión para aspirar a ser diputado.

En «un debito di seimila lire che il principino aveva garentito con una cambiale rinnovata parecchie volte di quattro in quattro mesi; il creditore, volen-

12. DISC: «Sottoporre un malato alle cure mediche necessarie per guarirlo, diagnosticare una malattia, individuare e mettere in atto la terapia atta a debellarla».

13. N 164: «un cierto dolor en el lado, al que en principio no dio importancia».

14. N 724: "Le había prodigado sus cuidados, le había demostrado un cariño fraternal, se había valido del ascendiente que tenía sobre él para curarlo». 
do esser soddisfatto e profittando della clausola del giovinotto, scriveva al padre» (II, VIII; p. 456), se traduce que «el acreedor quería cobrar y aprovechando la clausura del joven le escribía al padre» (M 483) cuando, sin embargo, la clausola se refiere a que el acreedor se apoyaba en que en dicha letra el muchacho había suscrito una clausola penale, es decir, una cláusula que establece el pago de una sanción por parte de quien viole las restantes cláusulas de un contrato: «aprovechándose de la cláusula firmada por el joven» (N 558).

A propósito de este asunto se desencadena una fuerte discusión entre el príncipe Giacomo y su hijo Consalvo, en la que el primero, como es normal, tutea al segundo y éste trata de usted $(L e i)$ a su padre. Al final de la discusión el príncipe le dice a su hijo: «esigo il rispetto che devi a tua madre», a lo cual el hijo responde con dureza: «Mia madre?... Mia madre è morta. Lei lo sa meglio di tutti» (II, VIII; p. 459-60), respuesta que M 487 traduce del siguiente modo: «¿Mi madre?... Mi madre está muerta. Ella lo sabe mejor que nadie»; sin embargo, coincido con N 562 en que la respuesta es en realidad: «Usted lo sabe mejor que nadie». Recordemos que Consalvo siempre sospechó de que la muerte de su madre Margherita — a finales de II, VI— había sido en cierta forma precipitada por su padre, que forzó a decidir a su mujer, ya enferma, a ponerse en camino desde el Belvedere, donde se había detectado el cólera, a Milo, en contra de lo que proponía Consalvo. Sobre la cuestión de los pronombres vuelvo a ocuparme más abajo.

El tío Eugenio se congracia con Raimondo e Isabella por interés recíproco, y de ello deriva que el primero «sinvitò tutti i giorni a colazione e a desinare» (II, IV; p. 360) a casa de los segundos, es decir, que use invitó todos los días a desayunar y a comer», motu proprio, algo diferente de «no pasó un día sin que se le invitara a desayunar y a comer» (M 385). Ahora bien, a propósito de la traducción alternativa que aquí propongo de este pasaje hay que observar que tampoco coincide con la de N 458: «se invitó a comer y a cenar todos los días». $\mathrm{Y}$ es que, en efecto, el nombre de las comidas y su traducción es una cuestión compleja e interesante, que merece un breve análisis.

De los cuatro nombres de comidas (o verbos que designan la acción de las mismas) empleados a lo largo de la novela - colazione, desinare, pranzo y cena (no consideramos un marginal refezione) - sólo cena (y cenare) resulta inequívoco en italiano y de traducción igualmente inequívoca en español, aunque su uso es escaso (solo tres cena y un cenare). En cuanto a pranzo, con quince casos, es término de equivalencia clara para $\mathrm{M}$, con el término comida-comer en todos los casos (es decir, como sinónimo de almuerzo-almor$z a r)$. En cuanto a N, aunque también opta en la mayoría de los casos por la comida-comer, hay dos casos en que traduce, a mi juicio sin fundamento, con cena-cenar:

Quando arrivò il Generale [Garibaldi], [Lodovico] gli andò incontro fino a piè dello scalone per dargli il benvenuto, lo guidò fino alle sue stanze, accompagnò ai loro alloggi gli aiutanti e presiedé il pranzo delle camicie rosse (II, III; p. 341). 
N 437-8: «Cuando llegó el general salió a su encuentro al pie de la escalinata para darle la bienvenida, lo condujo hasta sus aposentos, acompañó a los ayudantes a sus alojamientos, presidió la cena de los camisas rojas» (M 365: «la comida de los camisas rojas»).

Per tre giorni il palazzo restò ancora invaso dalla gente che veniva a congratularsi: una processione incessante dalle dieci del mattino a mezzanotte, con appena due ore di sosta per la colazione ed il pranzo (III, IX; p. 684).

N 796: El palacio siguió invadido todavía durante otros tres días por toda la gente que iba a felicitar al príncipe: desde las diez de la mañana hasta las doce de la noche era una procesión incesante, con un escaso intervalo de dos horas para comer y cenar (M 716: "para desayuno y comida»).

Hay un pasaje del que puede colegirse que De Roberto entendía por pranzo lo mismo que desinare, es decir, el almuerzo, ya que se emplean ambos términos para referirse a una misma comida concreta (aunque siempre puede objetarse que para De Roberto pranzo podía ser un término ambivalente, que significara en unos casos almuerzo y en otros cena, cosa que, no obstante, no parece probable):

E il domani Mazzarini, venuto a prenderlo, lo invitò a desinare con lui, trascinandolo al Morteo [...] Mazzarini stesso, prima di potersi sedere a tavola, dovette sbarazzarsi di quattro o cinque persone che lo aspettavano, e per tutta la durata del pranzo parlò della moltitudine delle sue faccende [...]. Finito il pranzo, un po' allegro, prese a braccio Consalvo [...]. Stanco [...] da quell'ignobile pranzo che aveva dovuto ingozzare per forza (III, II; p. 510)..$^{15}$

Las mayores divergencias a la hora de traducir se centran en los dos términos restantes, colazione y desinare. Según GDLI, desinare es "generalmente» sinónimo de pranzo y de pranzare, aunque también puede ser la cena; ${ }^{16} \mathrm{y}$, en efec-

15. M 540: «Al día siguiente Mazzarini pasó a recogerlo, lo invitó a comer con él y lo arrastró al Morteo [...] el mismo Mazzarini, antes de poder sentarse a la mesa, tuvo que desembarazarse de cuatro o cinco personas que lo esperaban y durante toda la comida habló de sus muchas ocupaciones [...] Terminada la comida, un tanto achispado, tomó del brazo a Consalvo [...]. Cansado [...] por esa comida vulgar que había tenido que tragarse a la fuerza». N 618-9: «Y al día siguiente Mazzarini, después de ir a buscarlo, lo invitó a comer con él, arrastrándolo hasta el restaurante Morteo [...] el mismo Mazzarini, antes de sentarse a comer, tuvo que deshacerse de cuatro o cinco personas que estaban esperándolo, y durante toda la comida estuvo hablando de sus múltiples ocupaciones [...] Al terminar la comida, un tanto alegre, cogió del brazo a Consalvo [...] Cansado [...] de la horrible comida que se había tenido que tragar a la fuerza».

16. "Consumare il principale pasto della giornata (generalmente verso il mezzogiorno); pranzare»; "Pasto principale della giornata che si consuma generalmente verso il mezzogiorno; pranzo". Tommaseo define desinare como «Il maggiore de' Pasti giornalieri che da alcuni suol farsi a mezzodì, e da altri, massimamente nelle città, più o meno verso sera", dejando abierta la posibilidad a que el desinare sea también la cena. También en DISC se antepone la acepción que lo hace sinónimo de almuerzo, aunque sin descartar la posibilidad de que sea la cena: «Consumare il pasto principale e più sostanzioso della giornata»; «Pasto di mezzogiorno, generalmente il più sostanzioso della giornata. / Pranzo, seconda colazione». En Zingarelli se igualan ambas acepciones: «Fare il pasto più sostanzioso della giornata, alla mattina $\mathrm{o}$ alla sera». 
to, de los veintiocho usos de este término que hay en la novela (sea como verbo que como sustantivo), en la mayoría de los casos ambos traductores han optado por el almuerzo, llamándolo comer o comida:

\begin{tabular}{lll}
\hline desinare & $\mathbf{M}$ & $\mathbf{N}$ \\
\hline 19 & comida-comer & comida-comer \\
\hline 4 & cena-cenar & comida-comer \\
\hline 4 & comida-comer & cena-cenar \\
\hline 1 & cena-cenar & cena-cenar \\
\hline
\end{tabular}

No obstante, como puede comprobarse, los casos en los que se traduce desinare con cena-cenar no son pocos, e incluso en un caso ambos traductores coinciden en esta opción, que el siguiente:

I, VII; p. 203: scappava via dopo colazione, tornava al finire del desinare per andar fuori un'altra volta fino a notte tarda.

M 221: se iba tan pronto como terminaba de desayunar, y regresaba al final de la cena para volver a salir entrada la noche.

N 291: se marchaba muy deprisa nada más comer, volvía cuando ya estaban terminando de cenar, para volver a salir hasta bien entrada la noche.

En mi opinión, todos los desinare que aparecen en la novela podrían traducirse siempre con comer-comida, incluso aquellos en los que, como éste último citado, aparece junto a colazione, ya que, a su vez, todos los usos de este término en la novela podrían traducirse con desayuno.

En efecto, no es menor es la disparidad de opciones para el término colazione. Como es sabido, este vocablo tiene dos acepciones principales: si se entiende prima colazione, es equivalente al español desayuno; si, por el contrario, se entiende seconda colazione, sería el almuerzo. El problema interpretativo nace desde el momento en que en ninguno de los doce casos en que es usado el término se especifica si es prima o seconda. El cuadro de traducciones es el siguiente:

\begin{tabular}{lll}
\hline colazione & $\mathrm{M}$ & $\mathrm{N}$ \\
\hline 5 & comida o almuerzo & comida o almuerzo \\
\hline 6 & desayuno & comida o almuerzo \\
\hline 1 & desayuno & desayuno \\
\hline
\end{tabular}

Tan sólo en un caso no hay lugar para la disparidad de interpretaciones, ya que se contrapone la colazione al pranzo: «Finito di pregare tornavano tutti 
nelle loro camere, facevano una colazione frugale perché il pranzo era a mezzogiorno, e ripassavano le lezioni» (I, VI; p. 180). ${ }^{17}$

Aludimos a continuación de forma más breve a otros casos que no requieren mucho comentario:

El otre es el odre o la bota de vino española, de manera que «ubbriacarvi come tanti otri» (II, III, p. 340) no es «emborracharos como otros muchos» (M 364), sino «emborracharos como pellejos» (N 437), algo equivalente a «emborracharos como cubas».

De Garibaldi se dice que es «un uomo che ha meriti distinti» (II, III, p. 346), traducido «un hombre, que reunirá otros méritos» (M 370). «Meriti distinti» es una fórmula (como «distinti saluti»), en la que distinto equivale a «especial, digno de distinción»: «distinguidos méritos» (N 443).

"la contessa, mentre faceva la gelosa, si divertiva anche lei in società! Non che fosse successo niente; in coscienza, questo non si poteva dire» (II, IV; p. 351), en donde lo subrayado ha sido traducido «No es que tuviera el menor éxito» (M 376). Cfr. N 449: «No es que hubiera pasado nada».

En «Se ci fossimo conosciuti prima, liberi entrambi!» (II, IV; p. 352), todo es una prótasis, mientras que la apódosis puede ser considerada la frase siguiente: «Come saremmo stati felicil»» «iSi nos hubiéramos conocido antes, ambos seríamos libres! ¡Qué dichosos habríamos sido!» (M 377). ${ }^{18}$

Scommettere es, principalmente, «apostar». Lucrezia asegura que la oposición de Giacomo a la relación entre Raimondo e Isabella no es debida a razones morales, sino económicas, y está tan segura de ello que incluso apostaría: «Per la morale? Per farsi pagare il suo appoggio! Scommettiamo? Io non ho dovuto pagargli il suo consenso al mio matrimonio? [...] Vedrete se sbaglio!» (II, IV; p. 360). Cfr. M 385: «¿Qué razones morales? ¡Para hacerse pagar su apoyo! ¿Que dejemos de meter cizaña? [...] ¡Verás si me equivoco!». En mi opinión, la traducción debería ser un simple «¿Apostamos algo?» o «¿Qué apostamos?». ${ }^{19}$

«Il Consiglio, il giorno prima, lo aveva eletto assessore» (III, IV; p. 554). Cfr. M 583: «El Consejo, al día siguiente, lo eligió concejal». N 662: «Un día antes había sido elegido delegado por el consejo».

«Tuo padre ha dichiarato che sposerai Michele, o nessuno. Non ho voluto dirtelo prima, credendo che egli si sarebbe piegato, ma tu lo conosci meglio di me» (III, v; p. 569). Cfr. M 599: «Tu padre ha dicho que te casarás con Michele o con nadie. No he querido decírtelo hasta ahora, creyendo que cederías, pero ya le conoces tú mejor que yo». N 679: «Tu padre ha declarado que o te casas con Michele o no te casas. No he querido decírtelo antes porque creía que se doblegaría, pero lo conoces mejor que yo».

17. M 196 «tomaban un desayuno frugal, porque la comida no era hasta mediodía»; N 266 "desayunaban frugalmente, porque la comida se servía a las doce».

18. N 450: «Si nos hubiéramos conocido antes, ¡cuando los dos estábamos libres! ¡Qué felices hubiéramos sido!...».

19. En N 458 no se ha traducido la palabra en cuestión, posiblemente por un descuido: «¿Por moral? ¡Para que le paguen su apoyo! ¿Es que yo no tuve que pagarle su consentimiento a mi boda?». 
«È vero che la ragazza aveva mostrato una grande arrendevolezza, e per questo ella la baciucchiava ogni quarto d'ora, in presenza della gente» (III, v; p. 581). Cfr. M 610: «Es verdad que la muchacha había dado muestras de gran docilidad, y por eso la buscaba cada cuarto de hora, en presencia de la gente». N 690: «Verdad es que la muchacha se había mostrado muy dócil y por eso la besuqueaba cada cinco minutos delante de la gente».

"L'operazioncella durò più che non si credesse e il principe dovè restare molti giorni a casa» (III, VII; p. 600). Cfr. M 630: «La pequeña operación duró menos de lo que cuesta decirlo, pero el príncipe tuvo que permanecer largos días en casa». N 710: «La pequeña operación duró mucho más de lo que en principio se pensaba y el príncipe tuvo que permanecer muchos días en casa sin salir».

«Il sindaco Consalvo fece da compare» (III, VII; p. 600). Cfr. M 630: «El alcalde Consalvo hizo lo posible por asistin». N 711: «El alcalde Consalvo fue el padrino».

En «Per l'allargamento del voto e per lo scrutinio di lista, non più le poche centinaia di elettori dello zio potevano mandarlo alla Camera: ce ne volevano migliaia» (III, VIII; p. 640), la preposición per equivale a «a causa de», «debido a»; es decir, "por» y no "para». Cfr. M 670: «Para la ampliación del voto y el escrutinio de la lista, los pocos cientos de electores de su tío no podían mandarlo a la Cámara: se requería un millar» y N 750: «Por la ampliación del voto y la elección en listas, ya no eran suficientes esos pocos centenares de electores de su tío, ahora eran necesarios algunos miles».

\section{II}

Un segundo grupo los errores de traducción son achacables a un descuido, bien de lectura del original o bien de escritura, y por tanto entran en un ámbito próximo al de las erratas. ${ }^{20}$ Por ejemplo «quattr'onze» (I, I; p. 29) es traducido con «cuarenta onzas» (M 32).

En «Il fidanzamento era stato perciò tutt'una guerra violenta fra cognato e cognata, tra zio e nipote ed anche tra madre e figlia» (I, III; p. 70), el tío es don Blasco y la nipote aludida es Chiara, que por tanto es «sobrina». Cfr. M 76: «Por eso el noviazgo había sido una auténtica guerra encarnizada entre cuñado y cuñada, entre tío y sobrino e incluso entre madre e hija». ${ }^{21}$

20. Señalo aquí las erratas que he encontrado en el texto: se habría el portillo (p. 10), lavapiati (11n), Moscaleri (44n), se había quedado con la cabizbaja (54), grababan (57), origines (113), Siclia (115n), primoénito (118), Giacinto [varias veces, en vez de Giacomo] (1216, 155-6, 279), Radetzsky (156n), aenaza (245), sólo están sólo los Giulente (286), Lodovido (365), hecer $(383,1.34)$, poniéndo (407), mientrastanto (452), no hacía sido crecer (464), Qué, (467, línea 28), lugarfue (516), [falta cerrar la interrogación] (529, línea 12), deinfluir (536), desgrácia (547), presatara (558), Progesista (617), cuatro hombre (637), Givovannino (641), las taquígrafos (702).

21. Un error similar es la traducción de «diede la mano da baciare alla nipote» (II, III; p. 321): «dio la mano a besar al sobrino» (M 345). 
Otro descuido lo encontramos en la traducción de «ma il più terribile di tutti fu il secondo Viceré, il grande Lopez Ximenes» (I, V; p. 169): «pero el más terrible de todos ellos fue el primer virrey, el gran López Jiménez» (M 184).

Las «cinquecent'onze all'anno» (III, vI; p. 597) que, según Eugenio, le legó su hermano don Blasco y le han sido negadas, se quedan simplemente en «quinientas onzas» (M 627).

El siguiente fragmento ha sido traducido de manera defectuosa: «Ferdinando, intento a mettere insieme un museo di storia naturale alle Ghiande, non s'era neppure informato di quel che avveniva; così, non solamente $i$ legatarii non avevano chiesto $i$ cont $i$; ma il principe, adducendo la mancanza di quattrini [...]» (I, VIII; p. 236). M 255: «Ferdinando, que trataba de reunir un museo de historia natural en las Ghiande, no sólo no le había exigido cuentas, sino que el mismo príncipe, alegando falta de liquidez [...]». ${ }^{22}$

También hay una omisión en la traducción de «Se io fossi morta?.. Se io non fossi nata?...»: (III, VII; p. 619): “¿Y si no hubiera nacido?...» (M 649), así como en el siguiente diálogo:

III, III, p. 521-2 M 551

«Io ne fui avvertito» affermò il principe.

«Ma a noi dicesti che non credevi ci fosse testamento..."

"Fui avvertito che si sentiva poco bene, non che avesse fatto testamento!»

«Avresti potuto farcelo sapere» ribatté donna Ferdinanda.
-Yo fui advertido - afirmó el príncipe.

- Pero a nosotros nos dijiste que creías que no había testamento...

-Podríais haberlo hecho saber — remachó doña Ferdinanda.

\section{N 629-30}

-Yo sí fui advertido — afirmó el príncipe.

- Pero a nosotros nos dijiste que no creías que existiera un testamento...

-iMe advirtieron de que no se encontraba bien, no de que hubiera hecho testamento! - Nos lo hubieras podido decir — le rebatió dama Ferdinanda.

Una última omisión que encontramos es la de una frase muy significativa, porque prácticamente resume el tema central de la novela: «ma egli disse allora una cosa che m'è parsa e mi pare molto giusta... Un tempo la potenza della nostra famiglia veniva dai re; ora viene dal popolo... La differenza è più di nome che di fatto" (III, IX; p. 689); "pero dijo entonces una cosa que me pareció y me parece muy cierta... La diferencia es más nominal que real» (M 722). ${ }^{23}$

En la grafía empleada en la novela, el plural de principe es principi y el de principio, principii (que en otras épocas había sido principj o principî̀ y moder-

22. Cfr. N 325: «Ferdinando, ocupado en montar un museo de historia natural en las Bellotas, ni siquiera se había enterado de lo que estaba sucediendo, de tal forma que los legatarios no sólo no habían pedido las cuentas, sino que el príncipe, además, aduciendo que no había dinero $[\ldots] »$.

23. Cfr. N 802: «pero añadió algo que me pareció y me sigue pareciendo razonable... En otra época la fuerza de nuestra familia procedía de los reyes, ahora procede del pueblo... La diferencia está más bien en el nombre que en los hechos...». 
namente se prefiere simplemente principi o principi). Por tanto, en la frase «I principi legittimi tornano tutti quanti» (I, VIII; p. 239) no se habla de principios (M 257: «Los principios legales han sido restablecidos»), sino de principes: «Están volviendo todos los príncipes legítimos» (N 327). ${ }^{24}$

En la traducción del siguiente pasaje ambos traductores han cometido descuidos. Dialogan Raimondo y su hermano Lodovico:

N 472-3

porque a Isabella se le impuso la boda y hay treinta testigos que probarán que es verdad...

- ¡Me consta! ¡Me consta!... Pero existe otra cuestión...

$$
\text { II, IV; p. } 375
$$

perché la volontá di Isabella fu violentata; trenta testimonii proveranno la verità..."

«Lo so! Lo so!... "rispose finalmente il Priore. "Io non t'avrei neppure ascoltato se non conoscessi che la ragione sta dalla vostra parte!!.

"Allora, posso fare assegnamento su te?» «Certo, certo!... Ma v'è un'altra quistione...

\section{400}

porque la voluntad de Isabella se vio forzada: treinta testigos están dispuestos a probar la verdad...

—iLo sé! ¡Lo sé!... —repuso finalmente el prior-. ¡No me habría molestado en escucharte si no supiera que tenéis a la religión de vuestra parte!

-Entonces, ¿puedo confiar en ti?

- iPor supuesto, por supuesto!... Pero hay otra cuestión...

Los fragmentos que reproducen la lectura de pasajes del Teatro genologico di Sicilia de Filadelfo Mugnòs ${ }^{25}$ constituyen, ciertamente, un interesante ejercicio de traducción y estilístico. Se trata de una obra del siglo XVII del que la ignorante solterona doña Ferdinanda recita algunos pasajes, para deleitarse con la nobleza de su estirpe:

Chiaramente per tvtti gli Hifpani genologifti fi fcorge, coi fvoi felici fvcceffi e con le occafioni debbite, qvale vna delle più antiche e fvblimi famiglie delli regni di Ualenza e d'Aragona la famiglia Vzeda, e per tvtto è uolgato effer ella fiffattamente cognominata dal nome, di vna fva terra detta la baronia di Vzeda, qvale alcanzò da qvei Re in ricompenfo dei fvoi fervigi et indi coi Trionfi della militia nel Svpremo Cielo delle glorie militari peruenne (I, III; p. 103).

24. Otros errores son los siguientes: «[Ferdinando] certi giorni neppur si levava» (II, IV; p. 364): «había días que ni siquiera se lavaba» (M 390). En II, v (p. 378), «Ed egli teneva il centro della sala» (M 403: «Y él ocupaba el centro de la calle»). «Ci vuole la sciocchezza di mia cognata» (II, v, p. 391), traducido «Se necesita ser tonta como mi hermana» (M 417). En III, II (p. 509) leemos: «faceva i suoi conti sulla morte del padre come sopra un avvenimento necesario alla propria felicità», traducido «hacía sus cábalas sobre la suerte de su padre como si de un acontecimiento necesario para su felicidad se tratase» (M 539).

25. Filadelfo Mugnòs, Teatro genologico delle famiglie dé regni di Sicilia Ultra e Citra, Palermo: P. Coppola, 1647-1660, 3 vols. (ed. facsímil: Bolonia: Forni, 2004). 
Como es sabido, la tipografía, hasta bien entrado el siglo XVIII, también en España, empleaba para la $s$ minúscula el símbolo $\int$, similar pero no igual a la f. ${ }^{26}$ Como señala A. Stussi, De Roberto manifestó explícitamente su voluntad de que su novela se imprimiera empleando, para las citas del Mugnòs, la fen lugar de la $s,{ }^{27}$ pero esta voluntad sólo fue respetada por los editores italianos hasta 1950, ya que a partir de entonces todas se olvidaron del símbolo en cuestión y recurrieron a la $f$ como sustituto de la $s$. En futuras traducciones españolas, por tanto, sugiero que se adopte también la $\int$ para estos pasajes: "Claramente deftaca la familia Vzeda, para todos los genealogiftas hifpanos, con fvs hechos infignes, etc...».

Al margen de ello, al traducir el citado pasaje, en mi opinión, ni M 113 ( "las glorias militares perennes») ni N 183 («las peruennes glorias militares») han transcrito bien la última palabra, que es pervenne - del verbo pervenire- $\mathrm{y}$ no perenne (ya que ésta última, en todo caso, debería ser perenni). La frase viene a decir: «y por ende con los Triunfos de la milicia alcanzó el Supremo Cielo de las glorias militares». ${ }^{28}$

En otros pasajes del Teatro genologico, leídos por el mismo personaje en I, V (p. 166-7), se narran las hazañas de algunos antepasados Uzeda (transcribo según la voluntad de De Roberto): «andò folo il Re appreflo vn groffo Cinghiale, il qvale aftvtamente fi trattenne nel corfo, ma perché il cauallo del Re fvriofamente di fopra gli correua, nel paffar impedito da quello, cafcò con

26. «La historia de la minúscula manuscrita se resume en dos formas, la típica sigual que la de imprenta o con su variante ligada y la ese alta $\int$, procedente de la escritura francesa, cuyo parecido con la $f(\mathrm{efe})$ ha provocado alguna que otra confusión, contra las que prevenía Ángel Rosenblat: "Hemos encontrado libros enteros de documentos que transcribe con $f$ toda $s$ larga, sin que al editor se le haya ocurrido vacilar o dudar un instante. Hay quien llama a esa $s$ larga 'la ese-efe'. Y hasta hemos oído a uno de esos historiadores, profesor universitario, decir a sus alumnos que antiguamente se pronunciaba así: Nueftro Feñor Jefucrifto"” (G. Salvador; J. R. Lodares, Historia de las letras, Madrid: Espasa-Calpe, 1996, p. 199).

27. «Nel terzo e nel quinto capitolo dei Vicerè ho qualche passaggio dove si citano antichi testi di quelli dove la $s$ avea la forma della $f$ ma senza lineetta. Per un certo mio effetto, è necessario che tutte le $s$ di queste citazioni siano della forma dell'effe senza linea; ma questo segno non si trova nelle tipografie. Ti prego dunque di persuadere Carlino che faccia fondere una trentina di queste $f$ : la spesa è di pochi soldi, e non credo che farà difficoltà [...] Bisognerà dunque che Carlino si persuada della necessità della fusione: ripeto, la spesa è una cosa ridicola, tanto tenue che non vale neppure la pena che io offra di sostenerla; ma se questo è necessario per ottenere l'intento, dirai che la mettano a mio carico» (carta a F. Di Giorgi, 18nov-1893; cito de A. STUSSI, "Esse, non effe! (nel centenario dei Viceré)", Italianistica, n. XXIII, 2-3, 1994, p. 513-14, p. 513). A propósito de la necesidad de emplear la $\int$ y no la f, Stussi también hace otra atinada observación: De Roberto precisa que Ferdinanda «leggeva effettivamente uolgato, peruenne», es decir, que confundía la $v$ con la $u$, pero no la $s$ con la $f$ : «era ovvio che donna Ferdinanda avesse familiarità con quelle esse lunghe e le leggesse senza confonderle con le effe» (ibid., p. 514). Por el contrario, cuando Consalvo, hablando con Ferdinanda en el último capítulo de la novela, imagina qué diría Mugnòs si viviera sobre los Uzeda del siglo XIX, De Roberto sí precisa que Consalvo "pronunciò $f$ la s» (III, IX; p. 691), porque al muchacho la tipografía antigua no le resulta familiar.

28. En la edición de la novela a cargo de C. A. MADRIGNANI, cit., puede leerse, efectivamente, la transcripción "pervenne». 
tvtto il Re in vn fafcio per terra, il qvale reftò con vna gamba di fotto il cauallo [...]». Es decir, que el caballo cayó al suelo «in un fascio», «todo vno con el Rei» (N 251), y no «in un sasso», traducido "el Rey dio con una piedra del suelo» (M 181).

\section{III}

En toda traducción se plantea la necesidad de adoptar previamente criterios que doten de coherencia al resultado final. Aunque, como es evidente, no siempre que aparezca una misma palabra ésta debe traducirse igual, ya que los contextos - como hemos visto a propósito de paese — pueden y deben condicionar su significado, en la medida de lo posible es conveniente buscar la homogeneidad.

En I, IX, al final de la primera parte de la novela, leemos un pasaje que contiene un término y una expresión que en el desarrollo posterior de la novela serán recurrentes y de una cierta relevancia: «e il principe, visto nel crocchio un jettatore, impallidiva mormorando: "Salute a noi, Salute a noi!"” (p. 285). En una peculiar manifestación de la locura que caracteriza a todos los miembros de la familia, el príncipe Giacomo muestra una patológica aversión hacia las personas que a él le parecen personas portadoras de mal agüero, y llega a considerar jettatore a su propio hijo Consalvo y a evitar llamarlo por su nombre, sustituyéndolo precisamente por Salut'a noi.

M 306: y el príncipe, al ver en el grupo a un pájaro de mal agüero, palideció mientras murmuraba: “QQue Dios nos asista! ¡Que Dios nos asista!».

N 377: y el príncipe, que había visto a un gafe en medio de la muchedumbre, se ponía pálido a la vez que murmuraba: «iTengamos salud! ;Tengamos salud!».

Ambas traducciones son muy válidas, ${ }^{29}$ pero veamos en qué medida los traductores han adoptado un criterio global para toda la novela. Jettatore había aparecido ya en el primer capítulo de la novela: «don Casimiro [...] fin a tre gior-

29. Es obligado recordar el artículo de L. SCIASCIA, «Jettatura, jettatore» (trad. española en El País, 4-sep-1988), del que no me resisto a extraer algunos fragmentos: «[la iettatura es una] antigua creencia de las regiones meridionales de Italia; pero cada vez más italiana, cada vez más de todas las regiones de Italia. Cabe esperar que no atraviese montes y mares, si ya no lo ha hecho. A la luz de los diccionarios, no parece que puedan traducirse al francés, inglés o español los sustantivos italianos iettatura y iettatore. Traducibles, quiero decir, con un solo sustantivo. Y en efecto, tanto en francés e inglés o español son necesarias frases para traducir ambas palabras italianas. Y si no existe la palabra, hay razones para creer que no existe la cosa. En los pequeños diccionarios de que dispongo, el francés traduce iettatore como jeteur de sort; el inglés, como bearer of ill-luck; el español, como persona de mal agüero. [...] Creo que a las palabras iettatura y iettatore les dieron ciudadanía en lengua italiana dos pequeños libros publicados en Nápoles en el siglo pasado. El primero, de Michele Arditi, arqueólogo, se titula Il fascino e l'amuleto contro il fascino presso gli antichi (El hechizo y el amuleto contra el hechizo entre los antiguos). El segundo es Cicalata sul fascino volgarmente detto iettatura (Charla sobre el embrujo vulgarmente llamado jettatura), de Nicola Valetta, libro que en la portada tiene un retrato de Valetta como para que uno piense en aquel personaje de Pirandello con- 
ni addietro era stato lavapiatti di casa Francalanza, ma fin da quando la principessa era andata in campagna, il principe non l'aveva più ricevuto, credendolo jettatore» (I, I; p. 31), un pasaje en el que M 35 ya había optado por "pájaro de mal agüero» mientras que N 104 por «cenizo» (a mi juicio, mejor que "gafe»). Sin embargo, en los nueve casos siguientes de toda la novela, $\mathrm{M}$ alternará su acertada elección de "pájaro de mal agüero» (cuatro casos) con «malasombra» (cinco), mientras que $\mathrm{N}$ se decide definitivamente por "cenizo», excepto un caso en el que opta por «echador de mal ojo» (N 534). El "potere jettatorio» (III, IV; p. 554), es traducido por M 584 con "poder de mal agüero» y por N 662 con "poder de echar mal de ojo".

En cuanto a la expresión-nombre Salut'a noi, que aparece una docena de veces en la novela, es uniformemente traducida por $\mathrm{N}$ con el ya mencionado «Tengamos salud», mientras que $\mathrm{M}$ alterna la forma ya citada, como opción principal, con otras dos:

Era morta, invece, —salut'a noi.- qualche mese dopo il matrimonio del conte e di donna Isabella (II, v; p. 381).

¡Estaba, en cambio, muerta - Jesús mio — a los pocos meses de la boda legal (M 407).

se Baldassarre o qualcuno dei lavapiatti o della servitù alludeva al principino, egli esclamava, afferrando l'ignobile amuleto, tenendolo stretto come in procinto di naufragare: «Salute a noi!... Salute a noi.» e ingiungeva alle persone di tacere (III, IV; p. 544).

si Baldassarre o cualquiera de los fregaplatos o de la servidumbre aludía al principito, él exclamaba, aferrando el innoble amuleto, que sostenía agarrado como si estuviese a punto de zozobrar: «Dios nos libre!... ¡Dios nos libre!..." y mandaba callar a las personas (M 574).

Un componente del texto más complejo de traducir, por su omnipresencia en toda la novela, es el relativo a los pronombres alocutivos. Los personajes de la novela se dirigen unos a otros según un estricto código formado por tres nive-

siderado como un jettatore y que quería que se le considerara un jettatore. [...] Sin embargo, se debe justamente a un lingüista napolitano, Basilio Puoti, la definición más razonable y precisa de la palabra iettatura: «Mal que falsamente, se supone provenir de personas a quienes se cree naturalmente maléficas e, incluso, a veces, deseosas de hacer daño». Más inefable y casi irritante como para creerla es, en cambio, la definición de Tommaseo en el Dizionario dei sinonimi: "Jettatore es quien, particularmente en Nápoles, se cree portador, con la presencia o las palabras, de desgracia y molestias; especie de brujo inocente y pasivo. Jettatore se nace como poeta [...] Sobre esta creencia necia y malvada, Pirandello escribió en 1911 una novela que luego convirtió en comedia: La patente». El traductor del artículo, C. Scavino, apostilla: «La palabra italiana jettatore podría también traducirse en España como gafe, y en Río de la Plata (Argentina) y Uruguay existe la misma palabra italiana, pero con i griega, yettatore, aunque en esta ocasión el autor hace referencia a la existencia de la frase mal de ojo para traducirla. En el diccionario de Julio Casares leemos: Gafe: m. fam. Cenizo, persona que trae a otras suerte adversa. En el diccionario enciclopédico Pequeño Larousse leemos: Yeta: f. Arg. Jettatura, mala suerte. Yetar: v. t. Arg. Producir mal de ojo. Jettatore: m. (pal. ital., pr. yetatore). Persona que tiene mal de ojo. Jettatura: f. Mal de ojo, entre los italianos». 
les de tratamiento: un nivel de reverencia (Vostra Eccellenza), un nivel de formalidad (voi) y un nivel de confianza $(t u)$. El nivel de reverencia está reservado para dirigirse a los miembros de la familia Uzeda, en primer lugar, por parte de la servidumbre. En segundo lugar, también es empleado por los propios Uzeda para dirigirse a otro pariente de una generación superior, aunque en este caso no es visto como un deber, sino como un derecho. Por otra parte, como el $V$. E. conlleva los pronombres y los verbos de $3^{\text {a }}$ persona singular, en ocasiones la agilidad de un dialógo lo reduce al Lei, como en la ya citada discusión entre el príncipe Giacomo y su hijo Consalvo, en la que éste le espeta: «Mia madre?... Mia madre è morta. Lei lo sa meglio di tutti» (II, VIII; p. 460). Incluso en un momento anterior de esta misma discusión, el hijo, en un gesto desafiante — y no de desconfianza — también subrayado por el narrador, había osado a dirigirse a su padre con el voi:

Consalvo piantò a un tratto gli occhi negli occhi del padre, guardandolo fisso, con un'espressione dura, come di sfida, e lasciato improvvisamente il lei:

«Che cosa volete?...» gli disse. «Avevo bisogno di danari... Me ne date tanti!... Li ho presi: voi che ne avete li pagherete...» (II, VIII; p. 457).

M 484: Consalvo clavó de repente los ojos en los de su padre, lo miró de hito en hito, con una expresión dura, como de desconfianza, y abandonando de pronto el «usted», dijo:

— ¿Qué quieres?... Necesitaba dinero... ¡Me das tanto!... Lo he cogido: tú que tienes lo pagarás.

N 559: Consalvo clavó de repente sus ojos en los de su padre, mirándolo fijamente, con una expresión muy dura, como desafiante, y dejando de repente a un lado el Usted:

—QQué queréis?... — - le dijo-. Necesitaba dinero... ¡Como me dais tanto!... Lo he cogido, vos que lo tenéis, pagadlo...

Los sobrinos y nietos políticos también emplean el $V$. E. para dirigirse a un Uzeda de una generación superior, como Isabella a don Blasco (p. 362), o Benedetto Giulente a Ferdinanda (p. 317). Sin embargo, un Uzeda no da ese tratamiento a un tío político: Consalvo no trata de V. E. a Benedetto Giulente, «ma del semplice voi» (II, VII; p. 446). Una variante del V. E. es el Vostra Paternità, que utilizan todos los Uzeda para dirigirse a un Uzeda religioso, don Blasco o don Lodovico.

En cuanto al voi, en efecto, es el pronombre de tratamiento que implica una relación formal y respetuosa, como en la conversación entre Gaspare Uzeda y Gaetano Palmi (II, I; p. 302-5). Raimondo e Isabella Fersa se tratan inicialmente de voi (p. 144), pero a partir de II, IV (p. 356) se tutean, y lo mismo sucede entre Giacomo y su prima Graziella (I, VII; p. 226: «da molti anni, da quando Giacomo aveva preso moglie, si davano del voi»).

Y por último, el tuteo, como es lógico, se emplea en toda relación de confianza. Los Uzeda lo emplean para dirigirse, por descontado, a la servidumbre, así como a un pariente de generación igual o posterior, al cónyuge. Cuando 
en vez del tuteo se utiliza el voi, el narrador suele hacerlo notar, como en una fase de la relación de Raimondo e Isabella «i parenti [...] udirono che dava a costei del voi [...] quel voi era già molto elocuente» (II, IX, p. 477); Los cuñados no Uzeda no tutean a un cuñado Uzeda: Isabella trata de voi a Ferdinando, y también Federico Villardita a Graziella (p. 263), incluso Giacomo trata de voi a su cuñada Matilde (p. 159). Fuera de los Uzeda, donde no existe el $V$. E., suele emplearse el tuteo incluso entre padre e hija, como Matilde con su padre el barón (p. 230).

A efectos de una traducción, obviamente, la elección que resulta más delicada es la del voi, toda vez que la traducción del Vostra Eccellenza y la del tuteo no plantea problemas.

En M 1994, el voi era traducido en la mayoría de los casos con vos, y en otros con usted, mientras que $\mathrm{M}$ emplea solamente usted. Incluyo a continuación también las soluciones de N, quien, como explica en p. 66n, traduce «el pronombre personal voi por vos, tú, $V d$. o vosotros, según las ocasiones, con objeto de reflejar el cuadro de las relaciones personales en Los Virreyes y el uso que del pronombre se hace en Sicilia, que varía según las zonas», aunque su opción mayoritaria para el voi es el propio voseo: ${ }^{30}$

I, II; p. 43: Quando credete, principe mio! Sono agli ordini vostri!...

M 1994, 54: ¡Cuando lo creáis conveniente, príncipe! ¡Estoy a vuestras órdenes!

M 49: ¡Cuando lo crea conveniente, príncipe! ¡Estoy a sus órdenes!

N 118: ¡Cuando creáis, príncipe mío! ¡Estoy a vuestras órdenes!

I, II, p. 52: Leggete tutto, di grazia

M 1994, 62: Léalo todo, se lo ruego

M 58: Léalo todo, por favor

N 128: Por favor, leedlo todo

En efecto, los pasajes en los que el narrador hace hincapié en el uso del voi por parte de algún personaje son traducidos en M 1994 con vos y en M con usted:

I, IV, p. 136: e smesso il tu, le dava del freddo voi

M 1994, 139: apeando el tú, le daba el frío tratamiento de vos

M 148: apeando el tú, le daba el frío tratamiento de usted;

N 218: habiendo dejado de tratarla de tú, la trataba fríamente de vos

I, VII, p. 226: si davano del voi

M 1994, 220: se trataban de vos

M 244: se trataban de usted

$\mathrm{N}$ 314: se trataban de vos

30. Efectivamente, son excepcionales los casos en los que $\mathrm{N}$ reduce el $v o i$ al $t u$, como en III, III (p. 522) cuando Lucrezia le espeta duramente a Garino "Voi state zitto!», traducido «Cállate» en N 630 («Usted cállese», en M 551); y también lo son aquellos en los que el voi es traducido con usted, como en II, VII (p. 446), donde se dice que Consalvo no trata de V. E. a su tío político Benedetto Giulente, «ma del semplice voi» (446), traducido «sino simplemente de Usted» (N 548), como en M 473. 
II, IX, p. 477: «i parenti [...] udirono che dava a costei del voi»

M 1994, 446: «los parientes [...] oyeron que trataba a ésta de vos»

M 504: «los parientes [...] oyeron que la trataba de usted»;

N 580: «la familia [...] oyeron cómo la trataba de vos»

III, II, p. 510: dandogli confidencialmente del voi

M 1994, 480: lo trataba en confianza de «vos»

M 539: lo trataba en confianza de «usted»

N 618: tratándolo confianzudamente de vos

El tratamiento Vostra Eccellenza era traducido en M 1994 con «vuestra excelencia», mientras que para $\mathrm{M}$ se ha optado por un menos recargado «su excelencia»; en ambos casos con minúscula, a diferencia de $\mathrm{N}$, que mimetiza el original con «Vuestra Excelencia»:

III, v; p. 583: Chiedo licenza a Vostra Eccellenza [...] Per niente, Eccellenza. Sono stato quarant'anni in casa di Vostra Eccellenza, ora me ne voglio andare. Vostra Eccellenza può tenermi per forza? In casa sua, Vostra Eccellenza comanda come gli pare e piace; chi le può dir nulla? Anch'io in casa mia sono padrone. Vostra Eccellenza può procurarsi un altro maestro di casa meglio di me.

M 613: Pido permiso a su excelencia. [...] Para nada, excelencia. He estado cuarenta años en casa de su excelencia, ahora deseo irme. ¿Puede retenerme por la fuerza, su excelencia? En su casa, su excelencia manda como le da la gana: ¿quién puede decirle nada?... También en mi casa soy yo el amo. Su excelencia puede conseguir otro mayordomo mejor que yo. ${ }^{31}$

N 692: Pido permiso a Vuestra Excelencia [...] Por nada, Excelencia. He estado cuarenta años en casa de Vuestra Excelencia, ahora quiero marcharme. ¿Acaso Vuestra Excelencia puede retenerme a la fuerza? En su casa Vuestra Excelencia manda como quiere y como le parece, jalguien puede decirle algo?... También yo soy el amo en mi casa. Vuestra Excelencia puede buscarse otro mayordomo mejor que yo.

La opción de $\mathrm{M}$ tiene el inconveniente de que el tratamiento de $2^{\mathrm{a}}$ persona coincide con el de $3^{a}$, ya que, no olvidemos, cuando un miembro de la servidumbre se refiere en $3^{\text {a }}$ persona a un Uzeda lo hace con Sua Eccellenza, como cuando un mensajero se dirige a don Blasco refieriéndole un recado de su hermano Gaspare: "arrivò a precipizio un altro messo da parte del duca. "Sua Eccellenza l'aspetta subito a casa... È affare urgentissimo"» (II, IX; p. 478).

31. Cfr. M 1994, 546: «Pido permiso a vuestra excelencia [...] Para nada, excelencia. He estado cuarenta años en casa de vuestra excelencia, ahora deseo irme. ¿Puede retenerme a la fuerza, vuestra excelencia? En su casa, vuestra excelencia manda como le da la gana: ¿quién puede decirle nada?... También en mi casa soy yo el amo. Vuestra excelencia puede conseguir otro mayordomo mejor que yo». 
En definitiva, no debemos olvidar que Los virreyes es una novela extensa, en la que, por tanto, los numerosos pasajes que hemos comentado tienen carácter puntual. Por otra parte, es innegable que una buena traducción debe serlo también en los más pequeños detalles, y que, en cualquier caso, las soluciones aportadas por Navarro, al menos en los pasajes analizados, son, sin excepción, más acertadas.

Concluyo con un último comentario. Para un italiano, "hablar en dialecto» tiene una precisa connotación sociolingüística e histórico-cultural, y más aún en la época de la Italia recién unificada, cuando la lingua — se entiende, por antonomasia, la italiana - era extraña para la mayoría de los italianos, cuya lengua materna era el dialetto, es decir, la lengua romance de su zona o región, en la que hablaban de manera espontánea y familiar, mientras que el italiano - fuera de Toscana- era portador de un carácter de oficialidad con el que pocos se sentían cómodos: «anche pe' più colti, parlare a mo' del dialetto è come adoperare la mano destra: parlare secondo le norme del buono italiano, è come adoperare la sinistra, per quanto si voglia educata». ${ }^{32}$ Esta situación era especialmente acusada en las zonas periféricas, como Sicilia, y tardaría aún muchos años en invertirse. El uso del dialecto, además, podía llegar a tener variedades muy atomizadas, propias de ámbitos muy restringidos, con formas peculiares de hablarlo, en el seno, incluso, de una familia. En la novela hay dos momentos en los que aflora esta cuestión: en un encuentro entre Giacomo y Raimondo "gli disse, non nel dialetto familiare, ma in lingua» (II, IV; p. 357), lo cual nos indica, por una parte, un dato importante: que los Uzeda hablaban habitualmente entre sí en dialetto (y por tanto, que los diálogos que hay entre ellos son traducidos por el narrador a la lingua); y en segundo lugar, que en esta ocasión Giacomo escoge deliberadamente la lingua para mostrarse frío y distante con su hermano, algo que refuerza con el gesto de no darle la mano, después de mucho tiempo sin verse. La traducción de M 382 viene a decir prácticamente lo contrario: «le dijo en el dialecto familiar, aunque en cristiano». ${ }^{33}$

El segundo pasaje es, en realidad, anterior: «Tutti sorrisero e il ghiaccio si ruppe. Smesso la dignità grave e il linguaggio fiorito dell'ambasceria, ognuno disse la sua, in dialetto, alla buona, per indurre il duca ad accettare» (I, IX; p. 269), traducido del siguiente modo: «[...] expuso su parecer en cristiano, sin ceremonias» (M 290). Recordemos que el dialecto, aunque asociable a un registro familiar y coloquial, no es un registro, sino una lengua a todos los efectos: «cada cual dijo lo que pensaba, en dialecto, sin cumplidos» (N 361, con interesante comentario y bibliografía en nota). Dicho de una manera más gráfica, con Weinrich, lo que diferencia al dialecto de la lengua es que «una lengua es un dialecto con un ejército y una armada». ${ }^{34}$

32. G. Finamore, Vocabolario dell'uso abruzzese, Lanciano: R. Carabba, 1880, p. IV-V (cito de B. Migliorini, Storia della lingua italiana [1960], Florencia: Sansoni, 1988, vol. II, p. 606).

33. Cfr. N 456: «se dirigió a él no en el dialecto familiar, sino en italiano».

34. Cito de S. Pinker [1994], El instinto del lenguaje. Cómo crea el lenguaje la mente. Madrid: Alianza, 1999, p. 29. 